See discussions, stats, and author profiles for this publication at: https://www.researchgate.net/publication/325371627

\title{
A multifunctional setup to record FTIR and UV-vis spectra of organic molecules and their photoproducts in astronomical ices
}

Article in Review of Scientific Instruments · May 2018

DOI: $10.1063 / 1.5027079$

CITATIONS

0

5 authors, including:

Vincent Kofman

Leiden University

3 PUBLICATIONS 3 CITATIONS

SEE PROFILE

Inge Loes ten Kate

Utrecht University

94 PUBLICATIONS 1,802 CITATIONS

SEE PROFILE

Some of the authors of this publication are also working on these related projects:

Project Carbon Chain Radicals View project

AQUEOUS ALTERATION OF POLYCYCLIC AROMATIC HYDROCARBONS View project
READS

85

Jordy Bouwman

Leiden University

39 PUBLICATIONS 453 CITATIONS

SEE PROFILE

H. Linnartz

Leiden University

297 PUBLICATIONS 4,459 CITATIONS

SEE PROFILE 
A multifunctional setup to record FTIR and UV-vis spectra of organic molecules and their photoproducts in astronomical ices

V. Kofman, M. J. A. Witlox, J. Bouwman, I. L. ten Kate, and H. Linnartz

Citation: Review of Scientific Instruments 89, 053111 (2018); doi: 10.1063/1.5027079

View online: https://doi.org/10.1063/1.5027079

View Table of Contents: http://aip.scitation.org/toc/rsi/89/5

Published by the American Institute of Physics 


\title{
A multifunctional setup to record FTIR and UV-vis spectra of organic molecules and their photoproducts in astronomical ices
}

\author{
V. Kofman, ${ }^{1,2, a)}$ M. J. A. Witlox, ${ }^{3}$ J. Bouwman, ${ }^{1}$ I. L. ten Kate, ${ }^{2}$ and H. Linnartz ${ }^{1}$ \\ ${ }^{1}$ Sackler Laboratory for Astrophysics, Leiden Observatory, Leiden University, P.O. Box 9513, \\ NL 2300 RA Leiden, The Netherlands \\ ${ }^{2}$ Department of Earth Sciences, Utrecht University, Budapestlaan 4, 3584 CD Utrecht, The Netherlands \\ ${ }^{3}$ Fine Mechanical Department, Leiden Institute for Physics Research (LION), Niels Bohrweg 2, \\ NL 2333 CA Leiden, The Netherlands
}

(Received 27 February 2018; accepted 30 April 2018; published online 25 May 2018)

\begin{abstract}
This article describes a new, multi-functional, high-vacuum ice setup that allows to record the in situ and real-time spectra of vacuum UV (VUV)-irradiated non-volatile molecules embedded in a lowtemperature $(10 \mathrm{~K})$ amorphous solid water environment. Three complementary diagnostic tools-UVvisible (UV-vis) and Fourier Transform Infrared (FTIR) spectroscopy and temperature-programmed desorption quadrupole mass spectrometry — can be used to simultaneously study the physical and chemical behavior of the organic molecules in the ice upon VUV irradiation. The setup is equipped with a temperature-controlled sublimation oven that enables the controlled homogeneous deposition of solid species such as amino acids, nucleobases, and polycyclic aromatic hydrocarbons (PAHs) in ice mixtures prepared from precursor gases and/or liquids. The resulting ice is photo-processed with a microwave discharge hydrogen lamp, generating VUV radiation with a spectral energy distribution representative for the interstellar medium. The characteristics, performance, and future potential of the system are discussed by describing three different applications. First, a new method is introduced, which uses broadband interference transmission fringes recorded during ice deposition, to determine the wavelength-dependent refractive index, $n_{\lambda}$, of amorphous solid water. This approach is also applicable to other solids, pure and mixed. Second, the UV-vis and FTIR spectroscopy of an VUV-irradiated triphenylene:water ice mixture is discussed, monitoring the ionization efficiency of PAHs in interstellar ice environments. The third and final example investigates the stability of solid glycine upon VUV irradiation by monitoring the formation of dissociation products in real time. Published by AIP Publishing. https://doi.org/10.1063/1.5027079
\end{abstract}

\section{INTRODUCTION}

Ice is an important reservoir of the molecular inventory in the colder and denser regions of the interstellar medium (ISM). When particle densities increase and temperatures are sufficiently low, atoms and molecules accrete and/or react on the surface of carbonaceous and silicate dust grains. This results in the formation of tens of monolayers comprising of different ice species. Space-based infrared observations with the Infrared Space Observatory (ISO) and Spitzer space telescopes have identified some 12-14 different ice constituents, ${ }^{1-3}$ including $\mathrm{H}_{2} \mathrm{O}, \mathrm{CO}, \mathrm{CO}_{2}, \mathrm{CH}_{3} \mathrm{OH}, \mathrm{CH}_{4}$, and $\mathrm{NH}_{3}$. The assignment of the astronomical data was possible with the help of infrared spectra recorded for pure and mixed ices under fully controlled laboratory conditions, i.e., in full dependency of the ice temperature and mixing ratios.

Many more species are expected to be present in the solid phase. It is generally accepted that chemical processes in the bulk and on the surface of interstellar ices are essential in facilitating the formation of both rather simple species such as $\mathrm{H}_{2} \mathrm{O}^{4,5}$ and $\mathrm{CH}_{3} \mathrm{OH}^{6}$ and more complex species, including small sugars and alcohol sugars like glycolaldehyde and glycerol. ${ }^{7-13}$ Laboratory experiments, indeed, confirmed that

a)Electronic mail: kofman@strw.leidenuniv.nl molecular complexity in an ice environment can be enhanced through (radiation-induced) radical recombinations, ${ }^{14,15}$ atom addition and abstraction reactions, ${ }^{13,16,17}$ or upon thermally induced processes during warm-up. ${ }^{18-21}$

Alternatively, larger species such as polycyclic aromatic hydrocarbons (PAHs), typically formed in the ejecta of dying stars, may also directly freeze out onto icy grains. Their characteristic vibrational gas phase features are observed as infrared emissions along many different lines of sight, but these vanish in regions where these species are expected to freeze out. ${ }^{22,23}$ It is therefore very likely that PAHs, like other volatiles, will accrete onto cold dust grains, resulting in a solid-state $\mathrm{PAH}$ reservoir in space. So far, unambiguous solid-state identifications of these larger species, both complex organic molecules and PAHs, are limited by lacking solid-state spectra or because broad and overlapping features do not allow to make unambiguous assignments. In fact, the limited number of solid-state identifications contrasts strongly with the more than 200 gas phase identifications of molecules in the ISM, of which many are thought to have a solid-state origin.

Since the 1970s, astrophysical ice mixtures have been studied in the laboratory, mostly using Fourier Transform Infrared (FTIR) spectroscopy. Over the decades, a special focus has been on the impact of energetic (VUV) photons on mixed ices originating from the interstellar radiation field, and 
it was shown that many organics can be found in the residues left over after irradiation, e.g., Refs. 14, 18, 24, and 25. To this day, infrared spectroscopy remains the go-to technique in laboratory ice astrochemistry, recording vibrational modes of the solid components. This enables one to assign typical absorption bands relatively easily but also comes with the problem that similar vibrational modes in different molecules exhibit about the same spectroscopic behavior; peak positions are not unique and solid-state features are typically broad, which complicates unambiguous identifications. Terminal and secondary functional groups are likely to have unique spectral signatures, but larger species are difficult to discern. This complicates the spectroscopic detection of molecules containing more than three carbon atoms. Another issue is that astronomical ice matrices, particularly water, strongly absorb in the IR. Therefore, in many laboratory IR ice studies, mostly pure or rather high concentrations of specific precursors have been used; this fails to represent realistic astronomical mixing ratios and will influence the chemical processes taking place. Optical, i.e., electronic ice spectroscopy offers a useful alternative.

Some 15 years ago, the first systematic studies of UV-vis ice absorption were reported. ${ }^{26-28}$ These come with a number of advantages over infrared studies. Water, the astronomically most abundant ice constituent, is transparent in this wavelength range, and this allows to use much lower concentrations of embedded species, particularly as transition strengths in the UV-vis can be orders of magnitudes stronger, further enabling the study of very dilute (and astronomically more relevant) mixtures. Another advantage of UV-vis spectroscopy is its higher time resolution: IR spectrometers are typically slow compared with their optical counterparts, which makes the monitoring of ongoing chemical processes in real-time difficult.

So far, most reported UV-vis ice experiments have been focusing on PAHs embedded in amorphous water environments (see, e.g., Refs. 26, 27, and 29-31). Many PAHs and their cations have large electronic oscillator strengths and absorb in the UV, visible or near IR, which facilitates such experiments, even for dilute mixtures. Studies have been reported previously of UV-vis spectra of PAHs and ionized PAHs embedded in water and ammonia environments. ${ }^{32}$ It was found that water ice decreases the ionization potential of the embedded PAHs, favoring the formation of cations, ${ }^{27}$ and that ammonia ice tends to enhance the formation of PAH anions. ${ }^{32}$ With higher water temperatures ( $40 \mathrm{~K}$ vs. $10-20 \mathrm{~K}$ ), the formation of hydrogenated and hydroxygenated PAHs was seen at the expense formation of the cation. ${ }^{30,33}$ It was also found that the concentration of the solute influences the ionization efficiency. ${ }^{34}$ An unfortunate disadvantage of UV-vis ice studies is that the assignment of electronic bands is harder to link to specific molecules than in the IR, and theoretical data are needed for assignments. Moreover, astronomical identifications of optical transitions of ices in space are currently still lacking.

The use of either IR or UV-vis spectroscopy to study interstellar ices clearly has its pros and cons. Combining both methods, therefore, offers an opportunity to benefit from the strengths of both methods and to link information obtained in one wavelength domain to findings in the other. Here, we describe a new setup capable of recording both UV-vis and FTIR of VUV photo-processed ice spectra in situ and quasi in real-time, monitoring ongoing dynamics in the ice. We demonstrate the use of UV-vis spectroscopy to track reaction products formed on a seconds time scale, and infrared spectroscopy to study the photostability of precursor species and photoinduced end-products. A complementary quadrupole mass spectrometer offers a further tool to identify (newly formed) ice constituents. Below we describe the experimental details and procedures and illustrate the potential of this setup on three selected examples: (i) the determination of the refractive index, $n_{\lambda}$, for amorphous solid water (ASW) at $10 \mathrm{~K}$ between 250 and $750 \mathrm{~nm}$; (ii) the VUV photochemistry of triphenylene $\left(\mathrm{C}_{18} \mathrm{H}_{12}\right)$ in $\mathrm{ASW}$ at $10 \mathrm{~K}$; and (iii) the formation of $\mathrm{HCN}$ and . $\mathrm{CN}$ from glycine upon VUV irradiation in an argon matrix at $10 \mathrm{~K}$ as a tool to monitor the photostability of frozen amino acids.

\section{SYSTEM DESCRIPTION}

Figure 1 shows a 3D schematic drawing, illustrating the concept of the new setup. The system comprises a central highvacuum chamber onto which the cold head of a closed-cycle helium refrigerator is mounted. Ices grown on a UV-vis and IR transparent window mounted on the tip of the cold finger can be irradiated with the VUV light from a microwave hydrogen discharge lamp and ice constituents, deposited, consumed, and formed are monitored using a broadband UV-vis and FTIR spectrometer as well as a quadrupole mass spectrometer that monitors ice constituents during thermal desorption.

\section{A. Main chamber}

The central part of the setup consists of an ISO 160 6-way cross piece, in which four additional CF 35 connections have

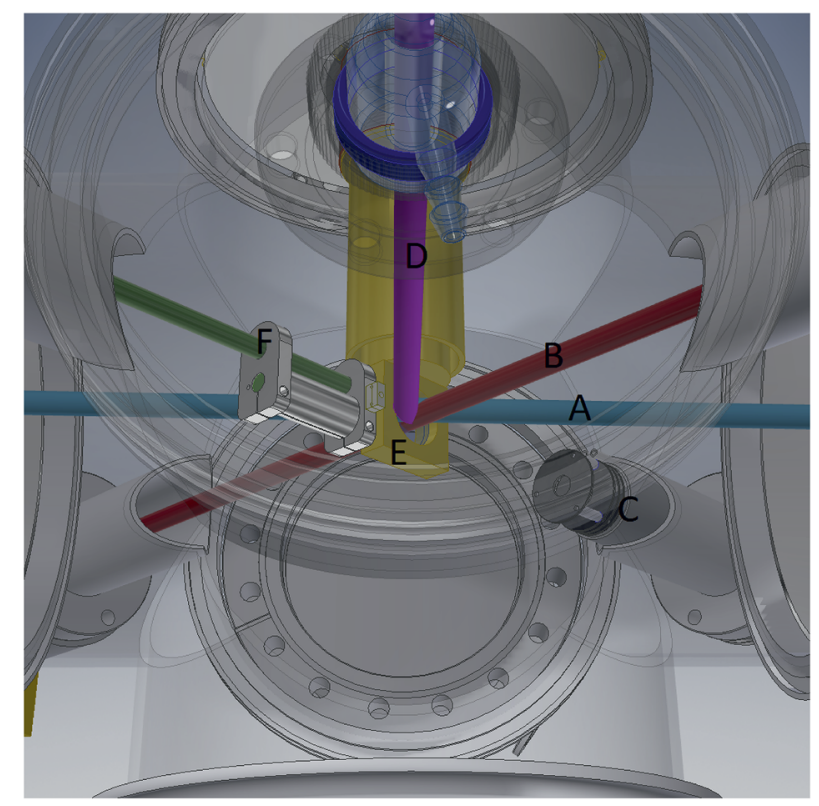

FIG. 1. 3D drawing of the interior of the vacuum system. The characters indicate the following: A. UV-vis path; B. Fourier Transform Infrared path; C. Quadrupole Mass Spectrometer; D. Vacuum ultraviolet light source; E. Cryostat with $\mathrm{BaF}_{2}$ window; F. Gas inlet and sublimation oven. 
been welded between the ISO ports, all directed towards the center of the cross piece. The vacuum chamber is mounted onto an optical table $(250 \times 125 \mathrm{~cm})$, which carries the two spectrometers and all necessary optical components.

One of the ISO ports supports a turbomolecular pump (Leybold 350 iX turbovac ISO 100, 290 1/s for $\mathrm{N}_{2}$ ), backed up by a two-stage $8 \mathrm{~m}^{3} / \mathrm{h}$ rotary pump, realizing a base pressure of the order of $10^{-8}$ mbar. To prevent oil from entering the system, a high-temperature cracking trap is placed on top of the prepump, which also reduces the load with potentially toxic species by thermally cracking these.

On the top flange of the vacuum chamber, the cold head of a closed-cycle helium cryostat is mounted (DE202NE, Advanced Research Systems, Inc.) via a turnable feedthrough. At the tip of the cold finger, $\mathrm{a} \mathrm{BaF}_{2}$ window is mounted, which is transparent from $140 \mathrm{~nm}$ to $16.7 \mu \mathrm{m}$. The sample temperature is controlled through resistive heating using a LakeShore 330 temperature controller. Temperatures between 10 and $325 \mathrm{~K}$ are accessible and measured using a factory-calibrated $\mathrm{Al}-\mathrm{Cr}$ thermocouple with an absolute precision better than $2 \mathrm{~K}$.

Ices are grown onto the cold sample through direct deposition at a normal angle, using a 1/4-in. Swagelok tube mounted $15 \mathrm{~mm}$ from the surface. A precision dosing valve (Pfeiffer EVN 116), equipped with a digital display for the setting, is used to control the flow of gas phase or liquid precursor species. A custom-designed and built oven, fully mounted inside the vacuum chamber, serves as a source to sublimate non-volatile molecules that can be co-deposited simultaneously with the ice. The oven is temperature-controlled and has a temperature precision better than $0.2{ }^{\circ} \mathrm{C}$, which is needed to guarantee a homogeneous deposition rate. The maximum temperature is roughly $150{ }^{\circ} \mathrm{C}$, which is more than sufficient to sublimate the amino acids and the small-to-medium PAHs targeted here. A 1/4-in. Swagelok tube, closed off at one end, is used as precursor reservoir. The reservoir slides into the oven and is removable, thus eliminating the need to detach the entire oven system from the vacuum chamber to add or replace the amino acids/PAHs. The orifice of the oven is positioned roughly $15 \mathrm{~mm}$ from the sample surface. In this configuration, dilution ratios can be realized as low as PAH: $\mathrm{H}_{2} \mathrm{O}=1: 10000$.

A microwave hydrogen discharge lamp (MHDL) is used as a VUV light source. These lamps have been used for decades to simulate interstellar radiation fields, ${ }^{35}$ peaking around Ly$\alpha(121 \mathrm{~nm})$ and $160 \mathrm{~nm}\left(\mathrm{H}_{2}\right.$ emission). In a series of recent papers, MDHL characteristics and their dependence on different settings have been discussed in detail. ${ }^{36-39}$ In our setup, a $150 \mathrm{~W}, 2450 \mathrm{MHz}$ Opthos microwave generator is used to run a plasma at 0.5 mbar $\mathrm{H}_{2}$ (grade 5.0) gas. The effective power of the generator is $100 \mathrm{~W}$. The resulting VUV flux amounts to $1-2 \times 10^{15}$ photons $/ \mathrm{cm}^{2} \mathrm{~s}$ at the sample surface, a value that is derived using oxygen/ozone actinometry. ${ }^{40,41}$ The MHDL is connected to the main chamber via a $\mathrm{MgF}_{2}$ window ( $25 \mathrm{~mm}$ diameter and $4 \mathrm{~mm}$ thickness). A tube is placed inside the chamber, which guides the light directly onto the sample. Stable and clean $\mathrm{H}_{2}$ flows in the MDHL are guaranteed using a small rotary pump that is equipped with an oil trap.

\section{B. Spectrometers}

The ice mixtures are studied using two different spectrometers, covering the wavelength domain associated with electronic and vibrational ice mode transitions.

UV-vis light (200-1100 nm) is generated using a deep UV xenon arc light source (150W LOT-QuantumDesign). The white light beam is spatially filtered by two subsequent pinholes, and it is transmitted through a $\mathrm{MgF}_{2}$ (25 mm diameter and $4 \mathrm{~mm}$ thick) window onto the ice substrate (positioned at $45^{\circ}$ with respect to the UV-vis beam). Light then exits the vacuum chamber through another $\mathrm{MgF}_{2}$ window and is focussed on the slit of an UV-vis spectrometer (Andor 303i Shamrock), equipped with a CCD (Andor iDus DV420 OE) responsive to photons between 200 and $1100 \mathrm{~nm}$. The available resolution depends on the used grating, where the spectral range coverage can be extended at the expense of a lower resolution. The maximum resolution accessible is $0.05 \mathrm{~nm}$, with a spectral coverage of roughly $50 \mathrm{~nm}$. In our ice experiments, a larger spectral range is favored over resolution. For the example experiments described later, spectra are recorded from 200 to $760 \mathrm{~nm}$ with a resolution of $0.56 \mathrm{~nm}$. To guarantee that the absolute wavelength calibration is well within any uncertainties for the used resolution, emission from the overhead fluorescence light, HeNe light, and emission from the xenon arc lamp are used to correct for grating offsets.

During a regular experiment, UV-vis spectra are taken every $4.3 \mathrm{~s}$, and such an averaged spectrum is the result of 120 individual spectra. The system allows for much higher time resolution, but for the experiments described below this is not necessary. Data taken after deposition are plotted as difference spectra with respect to the initially deposited sample.

Two CF 35 ports are used to transmit infrared light (2.5$16.7 \mu \mathrm{m} / 4000-600 \mathrm{~cm}^{-1}$ ) through the ice. An optical system consisting of gold-coated plano and concave mirrors is used to guide and focus the light from the external beam port of a Biorad FTS 3000 spectrometer onto the sample. A $90^{\circ}$ focusing mirror collects the IR light on a liquid $\mathrm{N}_{2}$ cooled mercurycadmium-tellurium (MCT) detector. The typical resolution used amounts to $2 \mathrm{~cm}^{-1}$. Higher resolution is possible, but this increases the measurement time per spectrum significantly, and is not required for the solid-state experiments such as those reported here. $\mathrm{ZnSe}$ windows are used as vacuum seals. The IR beam path is purged with dry and $\mathrm{CO}_{2}$ free air. A background spectrum is recorded before the deposition. In this way, the IR spectra reflect the entire sample processing (unlike the UV-vis spectra). Typically, 200 measurements are averaged, resulting in a time resolution of roughly $5 \mathrm{~min}$.

The setup has been constructed in such a way that both spectrometers can be used simultaneously (Fig. 1). Two different orientations are used. In position 1, the sample faces the deposition oven and is rotated $90^{\circ}$ with respect to the infrared beam path. This orientation is used for deposition and allows to perform UV-vis spectroscopy and VUV photo-processing at the same time, both at an angle of incidence of $45^{\circ}$. In position 2 (shown in Fig. 1), the sample faces the infrared beam, thus allowing transmission infrared spectroscopy at a normal angle, as well as the $\mathrm{UV}$-vis beam at a $45^{\circ}$ angle of incidence (this time passing the sample back to front). VUV processing 
TABLE I. Sample characteristics of the two triphenylene:water experiments.

\begin{tabular}{lcccccc}
\hline \hline & & & \multicolumn{3}{c}{ Column density (molecules cm $\left.{ }^{-2}\right)$} \\
\cline { 4 - 7 } Experiment & $d(\mathrm{~nm})$ & $\mathrm{C}_{18} \mathrm{H}_{12}: \mathrm{H}_{2} \mathrm{O}$ & UV-vis & $1497 \mathrm{~cm}^{-1}$ & $1437 \mathrm{~cm}^{-1}$ & $1253 \mathrm{~cm}^{-1}$ \\
\hline 1 & 247 & $1: 906$ & $7.6 \times 10^{14}$ & $1.5 \times 10^{15}$ & $2.7 \times 10^{15}$ & $2.0 \times 10^{15}$ \\
2 & 858 & $1: 3642$ & $7.0 \times 10^{14}$ & $1.5 \times 10^{15}$ & $2.3 \times 10^{15}$ & $2.1 \times 10^{15}$ \\
\hline \hline
\end{tabular}

is done at $45^{\circ}$. Additional deposition from the oven in this orientation is hindered, as the substrate faces the side of the copper sample holder.

Besides the two spectrometers, the setup is equipped with a quadrupole mass spectrometer (Pfeiffer Prisma Plus QMG 220) that is placed at one of the remaining CF 35 ports. This complementary tool allows us to link UV-vis and/or IR signals to species desorbing at specific temperatures, typically through their characteristic fragmentation patterns upon $70 \mathrm{eV}$ electron impact ionization, which are available from the NIST database. ${ }^{42}$ Mass spectra covering up to $300 \mathrm{~m} / \mathrm{z}$ are taken in bar graph mode, reporting the entire range of $\mathrm{m} / \mathrm{z}$ values at a resolution of $1 \mathrm{amu}$.

\section{Preparation and characterization of the sample}

Triphenylene:water samples are prepared by heating the oven to the sublimation temperature of triphenylene $\left(65^{\circ} \mathrm{C}\right)$, and two different ice thicknesses are realized by dosing water for $50 \mathrm{~min}$ at pressures of $0.5 \times 10^{-8} \mathrm{mbar}$ and $1.7 \times 10^{-8} \mathrm{mbar}$ above the base pressure (see Table I). Commercial triphenylene powder (roughly $5 \mathrm{mg}$ per fill, $98+\%$ purity) is used without further purification. For the water matrix, ultra-pure, milliQ-generated water is degassed in several freeze-pump-thaw cycles before use.

Matrix isolation experiments on glycine in argon are performed. Glycine is sublimated at an oven temperature set to $105^{\circ} \mathrm{C}$ and is deposited with argon (99.999\% purity) at a sample temperature of $10 \mathrm{~K}$. Prior to the sample deposition, the window is placed parallel with respect to the oven sample position, preventing triphenylene or glycine to deposit onto the sample before the desired temperature is established. Deposition is started by opening the dosing valve and rotating the sample so that it faces the sublimation oven and deposition line; i.e., we rotate the sample from position 2 to position 1. During deposition, UV-vis spectra are continuously taken. After completing the deposition, the sample is rotated and FTIR spectra are recorded. This allows us to study the ice growth during deposition with UV-vis, and after rotation the VUV-induced end products with both UV-vis and FTIR spectroscopy.

\section{RESULTS AND DISCUSSION}

\section{A. A new approach to measure ice thicknesses}

The sample thickness is an important parameter, as it has to be known to determine the concentration of the solutes under investigation. When ice is photo-processed by VUV light, the thickness also determines the photon penetration depth. Ice thicknesses are generally determined by recording the interference fringes that arise from a laser beam reflecting at the sample surface and at the top of the ice layer. The path length difference between the two light beams results in constructive and destructive interference signals, which change with increasing ice thickness. ${ }^{43-45}$ The intensity of the reflected light is measured with a photodiode. This results in a sinusoidal signal, where the period between the maxima $(m)$ is related to the thickness $d$ according to Eq. (1).

$$
d=\frac{m \times \lambda}{2 \times n_{\lambda} \times \cos \theta} .
$$

Here, $\lambda$ is the wavelength, $n_{\lambda}$ is the refractive index of the ice, and $\theta$ is the angle of incidence ( $45^{\circ}$ in our setup).

In our system, using UV-vis spectroscopy, an alternative approach is possible, which in addition offers the possibility to derive the full-wavelength-dependent refraction index of an (amorphous) ice. The applied principle is rather similar, but instead of looking for interference fringes by one external laser, fringes superimposed on the UV-vis transmission signal are used. This eliminates the need of an additional laser and photodiode, and instead the same broadband Xe arclight and spectrometer can be used, which are also needed for the spectroscopic investigation. An example of interference fringes seen at a number of selected wavelengths is shown in Fig. 2. For full investigation, fringes are recorded for hundreds of wavelengths at the same time. Clearly, the fringing is more pronounced for shorter wavelengths but visible over the full range. The figure also shows that over a set time interval for shorter wavelengths, the number of fringes, $m$, is larger than for longer wavelengths, in agreement with Eq. (1). This now allows, in principle, to derive ice thicknesses for any

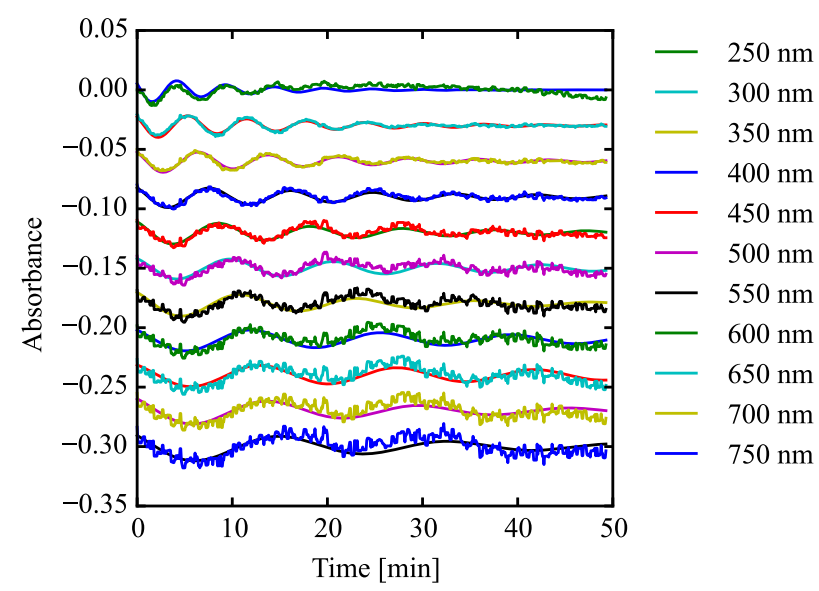

FIG. 2. Interference signal superimposed on the UV-vis signal as a function of time. A sinus multiplied with an exponential decay is fit to the signal and overplotted. The legend indicates the wavelength at which the signal is taken. 
wavelength, but as the refractive index is wavelengthdependent, $n_{\lambda}$ needs to be known first. For crystalline ice, these numbers are available from Warren and Brandt ${ }^{46}$ and show a variation with wavelength that gets more pronounced towards the UV. It should be noted that these values are for relatively high ice temperatures (in the astronomical context) and only measured at low resolution. For amorphous water ice, only a very limited number of $n_{\lambda}$ values is known and for practical reasons generally restricted to the $\mathrm{HeNe}$ wavelength, $632.8 \mathrm{~nm}$. Dohnálek et al. ${ }^{44}$ report a value of 1.285 for compact ASW with a density of $0.94 \mathrm{~g} / \mathrm{cm}^{3}$ at this wavelength. These values are not strongly temperature-dependent but depend mostly on porosity. ${ }^{44,47}$ Using this refractive index and assuming a density of $0.94 \mathrm{~g} / \mathrm{cm}^{3}$ for our ice, we calculate thicknesses of 247 and $858 \mathrm{~nm}$ for the thin and thick ice experiments, respectively, as listed in Table I. This now provides all information needed to derive $n_{\lambda}$. In a Python routine, the number of fringes is determined for all the recorded wavelengths, i.e., quasi continuous and for many more than the selected points shown in Fig. 2. Three separate experiments have been performed. The derived $n_{\lambda}$ curves are colored gray in Fig. 3. A fit through all data points, averaging the values from the individual measurements, results in a curve that is indicated in black and that clearly deviates from the values for crystalline ice, shown with the blue curve in Fig. 2. ${ }^{46}$ Typical deviations of 5\%-10\% exist when comparing the three experimental curves. This is acceptable, given the fact that these data are fully lacking in literature. The strength of this method is that it is relatively easy to determine $n_{\lambda}$ for a large wavelength domain — it takes less than a day to derive one curve—and that these numbers can be determined with a decent accuracy. As the method is generally applicable, this approach can be easily used to derive wavelength-dependent refractive indexes for other ices, pure and mixed. In fact, the method also will work

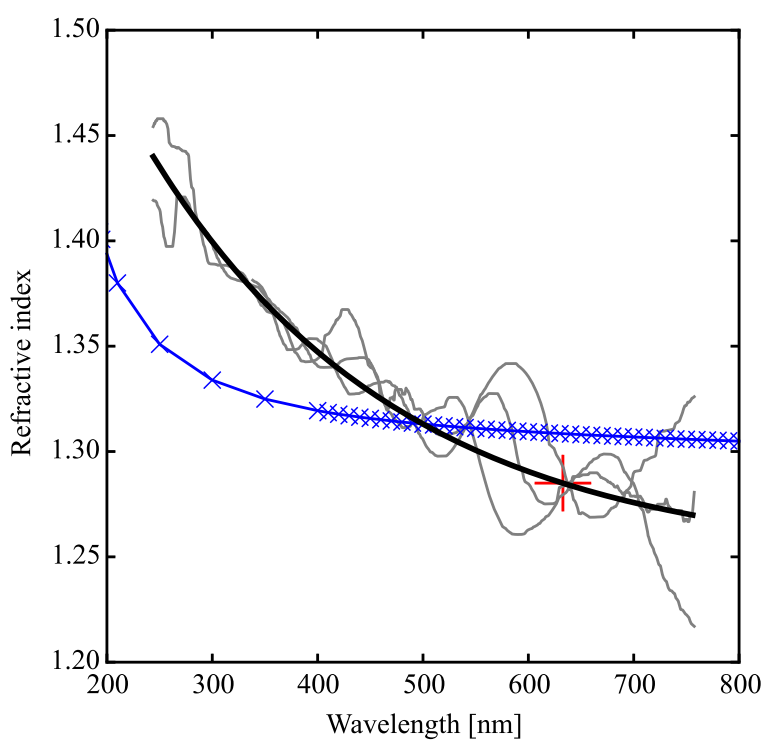

FIG. 3. Refractive indices, $n_{\lambda}$, as determined by the method described above for amorphous solid water at $10 \mathrm{~K}$, shown in black. The new values are based on three experiments, which are shown in gray. The $n_{\lambda}$ values of crystalline water ice are shown in blue ${ }^{46}$ the value reported in Dohnálek et al., ${ }^{44}$ which is used as a calibration point, is plotted as a red cross. fine for crystalline ices, which will allow a further fine-tuning. Work to use this method to provide $n_{\lambda}$ graphs of astronomically relevant ices is currently in preparation.

\section{B. Triphenylene:water ice mixtures; column densities and VUV photochemistry}

In order to determine the concentration of a solute, two parameters are needed, its abundance-which can be determined from the absorbance - and the ice thickness, as derived above. In the UV-vis, the abundance or column density can be quantified by integrating the area under an electronic transition $\left(\tau_{e l}\right)$ and dividing this by the oscillator strength $f$ and a factor as reported in Ref. 48:

$$
N_{e l}=\frac{\int_{v 1}^{v 2} \tau_{e l} d v}{8.85 \times 10^{-13} \times f} .
$$

In the experimental literature, absorption is often reported as the extinction coefficient, $\epsilon_{\max }$. To convert this to $f$, one uses ${ }^{49}$

$$
f=\frac{4.39 \times 10^{-9}}{n_{\lambda}} \int \epsilon d v .
$$

For triphenylene, a value of $\epsilon_{\max }=174000$ has been reported in Ref. 49.

Infrared transitions can be used in a similar way to quantify the column density:

$$
N_{v r}=2.303 \times \frac{\int_{v 1}^{v 2} \tau_{v i b} d v}{S},
$$

where the factor 2.303 converts the values from natural to base $10 \operatorname{logarithm}$, and $\int_{v 1}^{v 2} \tau_{v i b} d v$ is the integrated infrared band strength of the transition and $S$ is the transition strength in $\mathrm{cm}^{-1}$ per molecule.

Figure 4 shows the UV-vis spectrum of triphenylene in water for the thick ice. Except for a slight broadening this is identical to the thin ice spectrum. The spectral signatures of neutral triphenylene are observed at $255 \mathrm{~nm}$ for $S_{3} \leftarrow S_{0}$ and at $284 \mathrm{~nm}$ for $S_{2} \leftarrow S_{0}$. A weak series of transitions is found around $330 \mathrm{~nm} S_{1} \leftarrow S_{0}$, shown in more detail in the inset. These assignments are made based on existing matrix isolation studies. ${ }^{50,51}$ The reproducibility with which non-volatile species can be sublimated and deposited onto the substrate is illustrated by showing the maximum triphenylene absorption signal around $255 \mathrm{~nm}$ as function of time for the two ice thicknesses studied here. In the left panel of Fig. 5, these signals are shown as a function of time. Deposition starts at 0 and is in both experiments continued for about $50 \mathrm{~min}$. The high reproducibility is clear from the nearly identical growth curves. After the deposition, the sample is rotated, and a new reference spectrum is taken, which resets the signal to 0. After a short interval, the VUV irradiation is started, which results in the consumption of precursor material. Upon VUV irradiation, part of the neutral precursor is transferred into cationic triphenylene; the spectrum of the cation is presented in Fig. 6. This has been observed for other PAHs in the past $^{26,28}$ and the behavior with fluence is illustrated in the right part of Fig. 5. Clear triphenylene cation signals are found at 


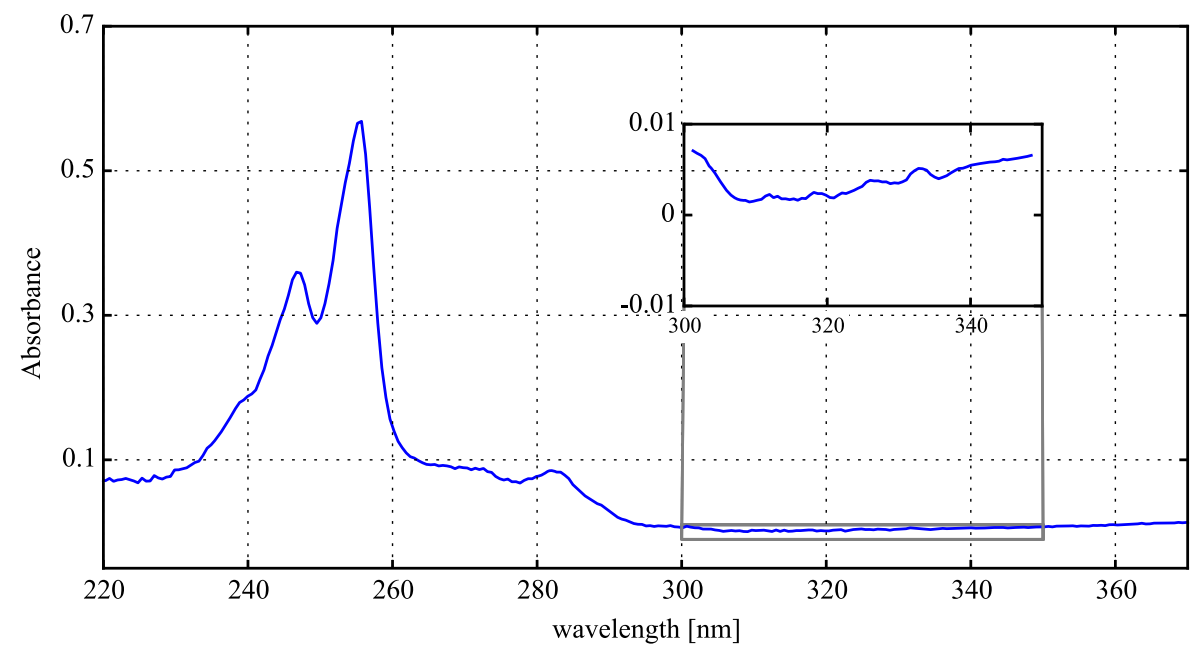

FIG. 4. UV-vis spectra of triphenylene in water. $S_{3} \leftarrow S_{0}$ is seen at $255 \mathrm{~nm}$, $S_{2} \leftarrow S_{0}$ at 285 , and the inset shows the weak $S_{1} \leftarrow S_{0}$ transitions. All of the transitions display strong vibroninic components. This spectrum is taken from experiment 2 .

$402 \mathrm{~nm}$, and broader features are found at lower wavelengths. Cation production works best for dilutions of the order of less than 1:1000 (PAH: $\left.\mathrm{H}_{2} \mathrm{O}\right) .{ }^{34}$ Here, the maximum ion column density is seen after roughly 15 min of irradiation. The formation yield of the cation is then roughly $30 \%$, taking a transition strength of the cation of $f=0.11 .^{52}$ Maximum yield is realized during the first few minutes of irradiation and is around $45 \%$.

For the thick ice, this process is less efficient than for the thin ice, which can be understood as in the latter case the VUV light reaches a larger fraction of the ice. In the right panel of Fig. 5, the integrated absorbance is shown, both for
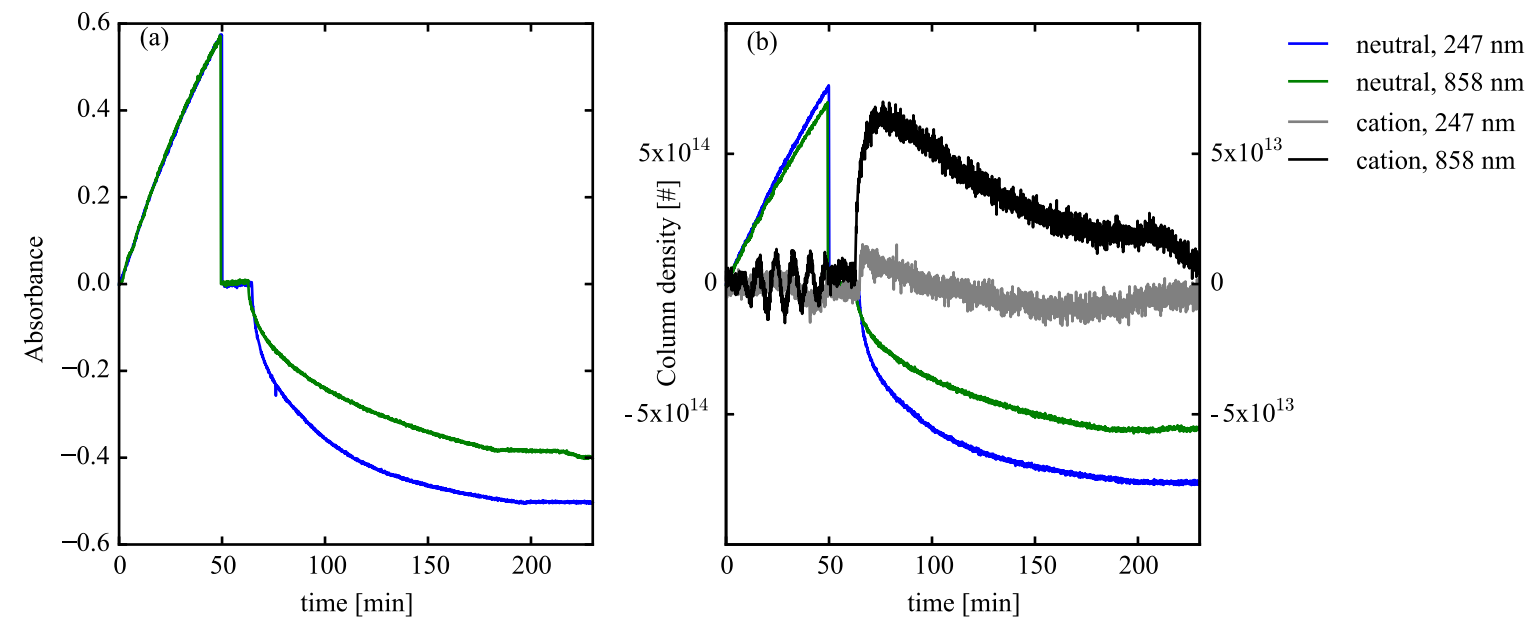

FIG. 5. (a) The time-dependent absorbance of the $\left(S_{3} \leftarrow S_{0}\right)$ transition of triphenylene recorded at a wavelength of $255 \mathrm{~nm}$ for the ices of two thicknesses (blue $247 \mathrm{~nm}$ and green $858 \mathrm{~nm}$ ). (b) Column densities for the experiment shown in (a) including also the VUV-induced ion signals. The cation column densities are plotted on the right y-axis. Negative features correspond with decreasing column density, and positive features reflect cation formation.

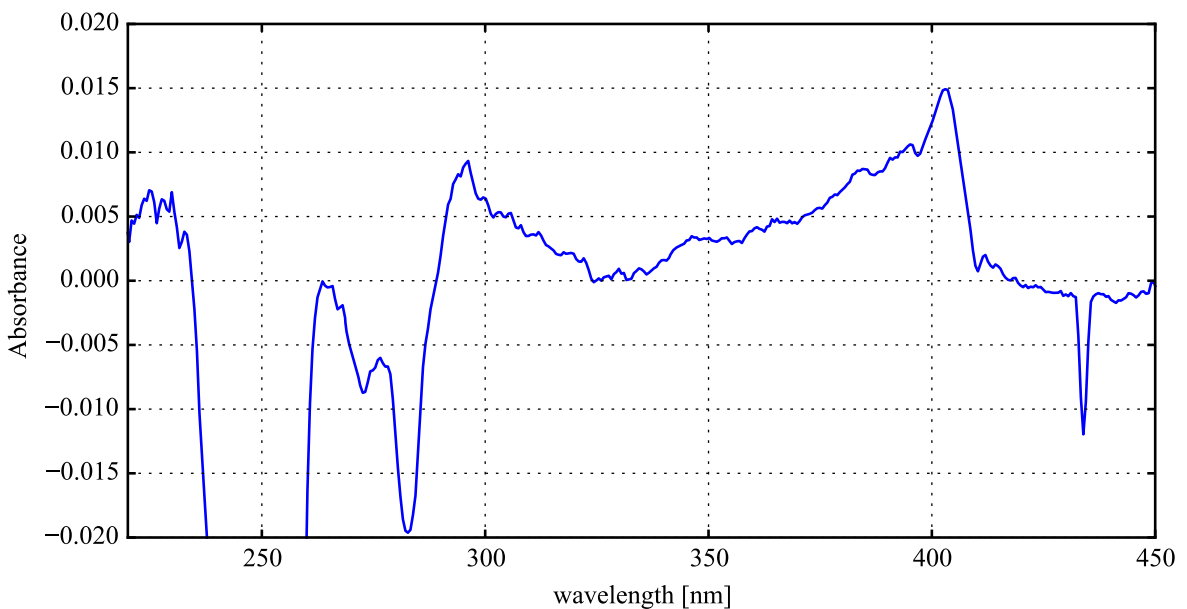

FIG. 6. UV-vis spectra of VUVirradiated triphenylene water mixture. Upon VUV irradiation, neutral $\mathrm{C}_{18} \mathrm{H}_{12}$ is destroyed, resulting in negative absorbance. Positive features are absorptions related to the cation. The strongest of these is seen at $402 \mathrm{~nm}$, having several broad features towards shorter wavelengths. 
the precursor material (that largely follows the curves in the left panel) and for the reaction product, the triphenylene cation. As the ionization efficiency is higher at lower concentrations, the cation formation is more effective in the thicker ice. Thus, although less neutral precursor material is consumed, a larger column density of cations are produced in the thicker ice with the lower triphenylene concentration.

Infrared spectra of triphenylene in water ice are shown in Fig. 7. The spectra are from the experiment where the concentration of triphenylene in water is roughly 1:900 (experiment 1). The spectrum illustrates that water signals largely dominate the spectrum, but the zoom-in shows that sufficient sensitivity is realized to also record the PAH bands. The theoretical band positions and band strengths are plotted as well, and this illustrates that the position and relative intensities reproduce well. ${ }^{53-55}$ The following bands are visible in the diluted water matrix: $1437 \mathrm{~cm}^{-1}$ most likely corresponding to two modes at $1440 \mathrm{~cm}^{-1}$ having a combined band strength of $7.8 \times 10^{-18} \mathrm{~cm}$ per molecule and similarly two transitions at $1253 \mathrm{~cm}^{-1}\left(1.1 \times 10^{-18} \mathrm{~cm}\right.$ per molecule $)$ and two at $1497 \mathrm{~cm}^{-1}\left(4.2 \times 10^{-18} \mathrm{~cm}\right.$ per molecule $)$.

Using the available literature values for triphenylene, we derive for the two experiments listed in Table I, column densities of 7.6 and $7.0 \times 10^{14}$ molecules $\mathrm{cm}^{-2}$ by integrating the UV-vis transition between 222 and $268 \mathrm{~nm}$. The equivalent exercise in the IR (at 1500,1440 , and $1253 \mathrm{~cm}^{-1}$ ) results in column densities that are approximately two to three times larger. It is very likely that this mismatch is due to an underestimate of the used band strengths as calculated by density functional theory (DFT) methods. This is in line with conclusions by Hardegree-Ullman et al. ${ }^{56}$ who find theoretical pyrene band strengths $50 \%$ larger than experimentally derived by Bouwman et al. ${ }^{57}$

Whereas the neutral precursor signals, though weak, are clearly visible, no IR features can be assigned to modes of the triphenylene cation. Based on the computed band strengths, we can estimate whether we should be able to. Assuming a maximum column density of roughly $6 \times 10^{13}$ molecules $\mathrm{cm}^{-2}$, cation signals should be visible when the band strengths from Langhoff ${ }^{53}$ are taken, but as discussed before such band strengths may have been overestimated. Clearly here UV-vis spectroscopy offers a powerful alternative.

\section{Photochemistry of glycine}

The recent detection of glycine, the smallest amino acid, on comet $67 \mathrm{P} / \mathrm{C}-\mathrm{G}^{59}$ has further strengthened the idea that complex organic molecules are formed in outer space on solid surfaces. Clearly, the formed glycine has been able to withstand the intense radiation in space. Understanding the photochemistry of this amino acid under VUV radiation is key in explaining its presence on comets and possibly other celestial objects. An experimental way of investigating the photostability of this amino acid, or other biologically relevant species, is by monitoring the time-dependent formation of fragments resulting from the photo-induced dissociation. We have studied this by VUV irradiation of glycine embedded in an argon matrix. Although not present in space, argon ice provides a chemically inert environment to study this process without the need to take into account other reactions due to a water matrix. Argon also provides a suitable host to trap produced radical species. Indeed upon VUV irradiation, and consistent with what has been reported in the literature (e.g. Ref. 60), $\mathrm{HCN}$ is formed from the fragmentation of frozen glycine. This can be understood by looking at the photo-degradation mechanism:

$$
\mathrm{NH}_{2} \mathrm{CH}_{2} \mathrm{COOH} \stackrel{h v}{\longrightarrow} \mathrm{CH}_{3} \mathrm{NH}_{2}+\mathrm{CO}_{2}
$$

$$
\mathrm{CH}_{3} \mathrm{NH}_{2} \stackrel{h v}{\longrightarrow} 2 \mathrm{H}_{2}+\mathrm{HCN}
$$

In Fig. 8(a), the integrated infrared absorbance of HCN is shown, which absorbs at $2091 \mathrm{~cm}^{-1}$. The assumed band strength is $5.1 \times 10^{-18} \mathrm{~cm}$ per molecule. ${ }^{58}$ Also shown in this figure are start and end times of the VUV processing, indicated by the magenta vertical lines. $\mathrm{HCN}$ is produced relatively slowly (the half-life time of glycine is in the order of a few minutes under these conditions), which is in agreement with the hypothesis that it is formed as a secondary product from the photo-degradation of the formed methylamine. In addition to the infrared detection of $\mathrm{HCN}$, we see the formation of the $\mathrm{CN}$ radical in the UV-vis, which absorbs between 376 and $384 \mathrm{~nm}$. Figure 8(b) shows the integrated band of $\cdot \mathrm{CN}$ in the UV-vis $\left(f=0.00320^{61}\right) . \cdot \mathrm{CN}$ is formed at the start of the VUV processing. A maximum column density of roughly

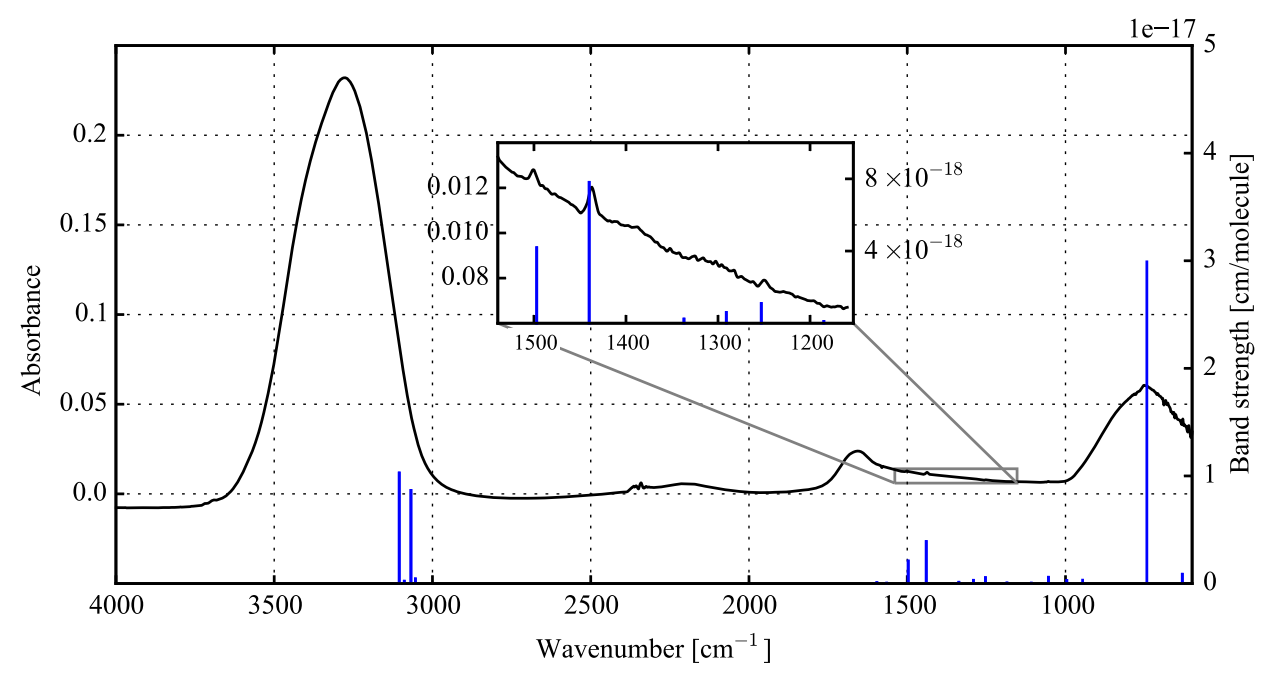

FIG. 7. Infrared spectrum of neutral triphenylene in water. Blue bars are the transitions of neutral triphenylene as predicted by Langhoff, ${ }^{53}$ corresponding to the $y$-axis on the right side. The inset shows a part of the fingerprint region. 


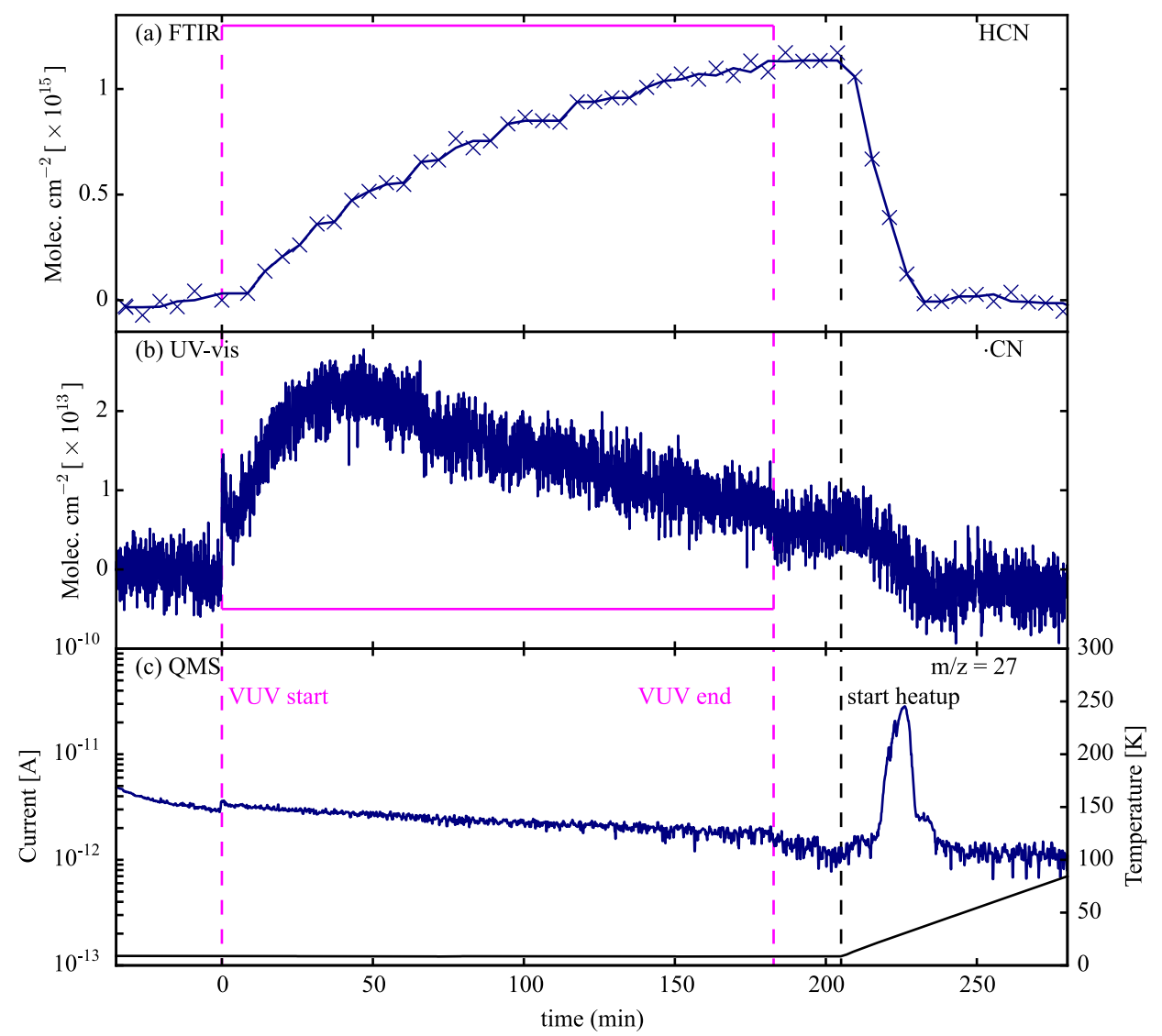

FIG. 8. Production of $\mathrm{HCN}$ as a function of time (i.e. fluence). VUV irradiation is indicated with the dashed vertical magenta lines $(t=0 \mathrm{~min}$ and $t=180 \mathrm{~min}$ ); the black dashed lines indicate the start of the ice heating ( $t=210 \mathrm{~min}$ ). (a) Column density of $\mathrm{HCN}$, derived from the transitions at $2091 \mathrm{~cm}^{-1}$. The reported band strength is $5.1 \times 10^{-18} \mathrm{~cm}$ per molecule. ${ }^{58}$ (b) Column density of $\cdot \mathrm{CN}$, derived from the transition at $380 \mathrm{~nm}$. Note that the integrated column density is much smaller than shown in (a). (c) $m / z=27$ (HCN) signal measured with the quadrupole mass spectrometer (QMS), which is the main signal seen upon electron impact of $\mathrm{HCN}$.
$2 \times 10^{13}$ molecules per $\mathrm{cm}^{2}$ is found after around $40 \mathrm{~min}$ and subsequently it is destroyed. In the period between the end of the irradiation and start of the heat-up, $\cdot \mathrm{CN}$ is stable.

The third panel (c) shows the results from parallel QMS experiments monitoring $\mathrm{m} / \mathrm{z}=27$, the dominant $\mathrm{HCN} \mathrm{m} / \mathrm{z}$ signal upon electron impact ionization. A heating ramp is started roughly $30 \mathrm{~min}$ after the end of the VUV processing. The temperature of the sample is also indicated in Fig. 8(c), placed on the secondary $\mathrm{y}$-axis (right side). The black line reports the temperature. A maximum of $\mathrm{m} / \mathrm{z}=27$ is seen coinciding with the disappearance of the respective species in (a) and (b). Panel (c) also shows the temperature axis of the temperature-programmed desorption (TPD).

As the measurements are performed simultaneously, the abundances of the products can be directly linked. The formation of - CN from VUV irradiated glycine has not been reported in the literature. From the formation kinetics, it appears to be formed earlier in the photo-degradation than HCN, enabling the possibility that $\mathrm{HCN}$ is formed from the hydrogenation of $\cdot \mathrm{CN}$. This would imply that the photochemical degradation of glycine results in different products depending on the environment, as the $\cdot \mathrm{CN}$ radical is highly reactive and could form different end-products. An extensive study, also looking at glycine in water, and looking at $\mathrm{CO}_{2}$ formation, is in preparation.

\section{ASTROPHYSICAL POTENTIAL}

Observations with the ISO and Spitzer Space telescopes have shown that icy dust grains are composed of different chemical components, layered and intimately mixed, amorphous and crystalline, porous and compact. So far, some twelve different ice constituents have been identified in the interstellar medium. With the upcoming launch of the James Webb Space Telescope, a new visible to infrared observatory (launch scheduled for 2020), it will become possible to investigate interstellar ices at spectral and spatial resolutions and sensitivities, orders of magnitude better than possible before. This will offer a view on the "Icy Universe" in unprecedented detail, also along lines of sight hard to observe up to now, e.g., towards the distant, crowded Galactic Center and the Magellanic Clouds. As icy dust grains are the formation place of complex organic molecules (COMs), which have been identified with the Atacama Large Millimeter Array in the gas phase, this also offers a tool to bridge the graingas-gap. Moreover, with the completion of the Extremely Large Telescope in the UV-NIR in 2024, also the recording of optical ice spectra towards embedded objects will become within reach. The full scientific impact of these observations, however, only will be realized when astronomical observations and astrochemical models can make use of precise laboratory ice data, ranging from spectroscopic data to wavelength-dependent refractive indices and recorded for known compositions, mixing ratios, temperature, porosity levels, and other parameters.

The setup described here offers this opportunity. It is expected that the number of solid-state identifications will quickly increase. This information and combined with gas phase detections of larger species in the ISM will allow to learn more on the solid-state processes at play. Also here the present setup can contribute by visualizing the chemical processes taking place. 
In this paper, the potential to study the behavior of PAHs and glycine upon VUV irradiation in cryogenic matrices has been illustrated. In the recent past, much focus has been put onto so called "GRAND PAHs," large polycyclic aromatic hydrocarbons with typically more than 40-50 C-atoms. Their presence in space has been explicitly linked to the observation of the aromatic infrared bands, ${ }^{22}$ and several studies on their photo-fragmentation behavior have been recently reported. Compact and strongly bound smaller PAHs may be relevant as well; triphenylene $\left(\mathrm{C}_{18} \mathrm{H}_{12}\right)$ belongs to this category as it is fully benzenoid. Other COMs are observed in the gas phase as well, and as only solid-state pathways offer the efficiencies to explain their formation, it is clear that experiments are needed to characterize both their spectroscopic and dynamical features, like for the glycine discussed here.

\section{ACKNOWLEDGMENTS}

Financial support through the NWO program PEPSci (Planetary and ExoPlanetary Science) and the ITN program EUROPAH is acknowledged. The authors thank Niels Ligterink, Ko-Ju Chuang, Sergio Ioppolo, and Sergio Pilling for input and discussions regarding the design of the setup. Raymond Koehler is thanked for designing and building the oven controller. This research has made use of NASA's Astrophysics Data System.

${ }^{1}$ E. L. Gibb, D. C. B. Whittet, A. C. A. Boogert, and A. G. G. M. Tielens, "Interstellar ice: The infrared space observatory legacy," Astrophys. J., Suppl. Ser. 151, 35-73 (2004).

${ }^{2}$ K. I. Öberg, A. C. A. Boogert, K. M. Pontoppidan, S. van den Broek, E. F. van Dishoeck, S. Bottinelli, G. A. Blake, and N. J. Evans II, "The Spitzer ice legacy: Ice evolution from cores to protostars," Astrophys. J. 740, 109 (2011).

${ }^{3}$ A. C. A. Boogert, P. A. Gerakines, and D. C. B. Whittet, "Observations of the icy universe," Annu. Rev. Astron. Astrophys. 53, 541-581 (2015).

${ }^{4}$ S. Ioppolo, H. M. Cuppen, C. Romanzin, E. F. van Dishoeck, and H. Linnartz, "Laboratory evidence for efficient water formation in interstellar ices," Astrophys. J. 686, 1474-1479 (2008).

${ }^{5}$ N. Miyauchi, H. Hidaka, T. Chigai, A. Nagaoka, N. Watanabe, and A. Kouchi, "Formation of hydrogen peroxide and water from the reaction of cold hydrogen atoms with solid oxygen at $10 \mathrm{~K}$," Chem. Phys. Lett. 456, 27-30 (2008).

${ }^{6}$ G. W. Fuchs, H. M. Cuppen, S. Ioppolo, C. Romanzin, S. E. Bisschop, S. Andersson, E. F. van Dishoeck, and H. Linnartz, "Hydrogenation reactions in interstellar $\mathrm{CO}$ ice analogues. A combined experimental/theoretical approach," Astron. Astrophys. 505, 629-639 (2009).

${ }^{7}$ S. Ioppolo, Y. van Boheemen, H. M. Cuppen, E. F. van Dishoeck, and $\mathrm{H}$. Linnartz, "Surface formation of $\mathrm{CO}_{2}$ ice at low temperatures," Mon. Not. R. Astron. Soc. 413, 2281-2287 (2011).

${ }^{8}$ E. F. van Dishoeck, E. Herbst, and D. A. Neufeld, "Interstellar water chemistry: From laboratory to observations," Chem. Rev. 113,9043-9085 (2013).

${ }^{9}$ E. A. Bergin and M. Tafalla, "Cold dark clouds: The initial conditions for star formation," Annu. Rev. Astron. Astrophys. 45, 339-396 (2007).

${ }^{10}$ R. T. Garrod, S. L. Widicus Weaver, and E. Herbst, "Complex chemistry in star-forming regions: An expanded gas-grain warm-up chemical model," Astrophys. J. 682, 283-302 (2008).

${ }^{11}$ E. Herbst and E. F. van Dishoeck, "Complex organic interstellar molecules," Annu. Rev. Astron. Astrophys. 47, 427-480 (2009).

${ }^{12}$ G. Fedoseev, K.-J. Chuang, S. Ioppolo, D. Qasim, E. F. van Dishoeck, and H. Linnartz, "Formation of glycerol through hydrogenation of $\mathrm{CO}$ ice under prestellar core conditions," Astrophys. J. 842, 52 (2017).

${ }^{13} \mathrm{H}$. Linnartz, S. Ioppolo, and G. Fedoseev, "Atom addition reactions in interstellar ice analogues,” Int. Rev. Phys. Chem. 34(2), 205-237 (2015).

${ }^{14}$ M. P. Bernstein, J. P. Dworkin, S. A. Sandford, G. W. Cooper, and L. J. Allamandola, "Racemic amino acids from the ultraviolet photolysis of interstellar ice analogues," Nature 416, 401-403 (2002).
${ }^{15}$ K. I. Öberg, R. T. Garrod, E. F. van Dishoeck, and H. Linnartz, "Formation rates of complex organics in UV irradiated $\mathrm{CH}_{3} \mathrm{OH}$-rich ices. I. Experiments," Astron. Astrophys. 504, 891-913 (2009).

${ }^{16}$ K.-J. Chuang, G. Fedoseev, S. Ioppolo, E. F. van Dishoeck, and H. Linnartz, "H-atom addition and abstraction reactions in mixed $\mathrm{CO}$, $\mathrm{H}_{2} \mathrm{CO}$ and $\mathrm{CH}_{3} \mathrm{OH}$ ices-An extended view on complex organic molecule formation," Mon. Not. R. Astron. Soc. 455, 1702-1712 (2016).

${ }^{17}$ K.-J. Chuang, G. Fedoseev, D. Qasim, S. Ioppolo, E. F. van Dishoeck, and H. Linnartz, "Production of complex organic molecules: H-atom addition versus UV irradiation," Mon. Not. R. Astron. Soc. 467, 2552-2565 (2017).

${ }^{18}$ G. M. Muñoz-Caro, U. J. Meierhenrich, W. A. Schutte, B. Barbier, A. Arcones Segovia, H. Rosenbauer, W. H.-P. Thiemann, A. Brack, and J. M. Greenberg, "Amino acids from ultraviolet irradiation of interstellar ice analogues," Nature 416, 403-406 (2002).

${ }^{19}$ T. Butscher, F. Duvernay, A. Rimola, M. Segado-Centellas, and T. Chiavassa, "Radical recombination in interstellar ices, a not so simple mechanism," Phys. Chem. Chem. Phys. 19, 2857-2866 (2017).

${ }^{20}$ M. Nuevo, J. H. Bredehöft, U. J. Meierhenrich, L. L. S. d'Hendecourt, and W. H.-P. Thiemann, "Urea, glycolic acid, and glycerol in an organic residue produced by ultraviolet irradiation of interstellar/pre-cometary ice analogs," Astrobiology 10, 245-256 (2010)

${ }^{21} \mathrm{P}$. de Marcellus, C. Meinert, I. Myrgorodska, L. Nahon, T. Buhse, L. L. S. d'Hendecourt, and U. J. Meierhenrich, "Aldehydes and sugars from evolved precometary ice analogs: Importance of ices in astrochemical and prebiotic evolution,” Proc. Natl. Acad. Sci. U. S. A. 112, 965-970 (2015).

${ }^{22}$ L. J. Allamandola, A. G. G. M. Tielens, and J. R. Barker, "Interstellar polycyclic aromatic hydrocarbons-The infrared emission bands, the excitation/emission mechanism, and the astrophysical implications," Astrophys. J., Suppl. Ser. 71, 733-775 (1989).

${ }^{23}$ A. G. G. M. Tielens, "The molecular universe," Rev. Mod. Phys. 85, 1021-1081 (2013).

${ }^{24}$ W. Hagen, L. J. Allamandola, and J. M. Greenberg, "Interstellar molecule formation in grain mantles-The laboratory analog experiments, results and implications," Astrophys. Space Sci. 65, 215-240 (1979).

${ }^{25}$ C. Meinert, I. Myrgorodska, P. de Marcellus, T. Buhse, L. Nahon, S. V. Hoffmann, L. L. S. d'Hendecourt, and U. J. Meierhenrich, "Ribose and related sugars from ultraviolet irradiation of interstellar ice analogs," Science 352, 208-212 (2016).

${ }^{26}$ M. S. Gudipati and L. J. Allamandola, "Facile generation and storage of polycyclic aromatic hydrocarbon ions in astrophysical ices," Astrophys. J. 596, L195-L198 (2003).

${ }^{27}$ M. S. Gudipati and L. J. Allamandola, "Polycyclic aromatic hydrocarbon ionization energy lowering in water ices," Astrophys. J. 615, L177-L180 (2004).

${ }^{28}$ M. S. Gudipati and L. J. Allamandola, "Unusual stability of polycyclic aromatic hydrocarbon radical cations in amorphous water ices up to $120 \mathrm{~K}$ : Astronomical implications," Astrophys. J. 638, 286-292 (2006).

${ }^{29}$ J. Bouwman, H. M. Cuppen, A. Bakker, L. J. Allamandola, and H. Linnartz, "Photochemistry of the PAH pyrene in water ice: The case for ion-mediated solid-state astrochemistry," Astron. Astrophys. 511, A33 (2010).

${ }^{30} \mathrm{Z}$. Guennoun, C. Aupetit, and J. Mascetti, "Photochemistry of coronene with water at $10 \mathrm{~K}$ : First tentative identification by infrared spectroscopy of oxygen containing coronene products," Phys. Chem. Chem. Phys. 13(16), 7340-7347 (2011).

${ }^{31}$ A. Lignell and M. S. Gudipati, "Mixing of the immiscible: Hydrocarbons in water-ice near the ice crystallization temperature," J. Phys. Chem. A 119, 2607-2613 (2015).

${ }^{32}$ S. H. Cuylle, E. D. Tenenbaum, J. Bouwman, H. Linnartz, and L. J. Allamandola, "Ly $\alpha$-induced charge effects of polycyclic aromatic hydrocarbons embedded in ammonia and ammonia:water ice," Mon. Not. R. Astron. Soc. 423, 1825-1830 (2012).

${ }^{33}$ A. M. Cook, A. Ricca, A. L. Mattioda, J. Bouwman, J. Roser, H. Linnartz, J. Bregman, and L. J. Allamandola, "Photochemistry of polycyclic aromatic hydrocarbons in cosmic water ice: The role of PAH ionization and concentration," Astrophys. J. 799, 14 (2015).

${ }^{34}$ S. H. Cuylle, L. J. Allamandola, and H. Linnartz, "Photochemistry of PAHs in cosmic water ice. The effect of concentration on UV-VIS spectroscopy and ionization efficiency," Astron. Astrophys. 562, A22 (2014).

${ }^{35} \mathrm{P}$. Warneck, "A microwave-powered hydrogen lamp for vacuum ultraviolet photochemical research," Appl. Opt. 1, 721-726 (1962).

${ }^{36}$ G. M. Muñoz-Caro and W. A. Schutte, "UV-photoprocessing of interstellar ice analogs: New infrared spectroscopic results," Astron. Astrophys. 412 121-132 (2003). 
${ }^{37}$ Y.-J. Chen, K.-J. Chuang, G. M. Muñoz-Caro, M. Nuevo, C.-C. Chu, T.-S. Yih, W.-H. Ip, and C.-Y. R. Wu, "Vacuum ultraviolet emission spectrum measurement of a microwave-discharge hydrogen-flow lamp in several configurations: Application to photodesorption of CO ice," Astrophys. J. 781, 15 (2014).

${ }^{38}$ N. F. W. Ligterink, D. M. Paardekooper, K.-J. Chuang, M. L. Both, G. A. Cruz-Diaz, J. H. van Helden, and H. Linnartz, "Controlling the emission profile of an $\mathrm{H}_{2}$ discharge lamp to simulate interstellar radiation fields," Astron. Astrophys. 584, A56 (2015).

${ }^{39}$ E. T. Es-sebbar, Y. Bénilan, N. Fray, H. Cottin, A. Jolly, and M.-C. Gazeau, "Optimization of a solar simulator for planetary-photochemical studies," Astrophys. J., Suppl. Ser. 218, 19 (2015).

${ }^{40} \mathrm{H}$. Cottin, M. H. Moore, and Y. Bénilan, "Photodestruction of relevant interstellar molecules in ice mixtures," Astrophys. J. 590, 874-881 (2003).

${ }^{41}$ D. Fulvio, A. C. Brieva, S. H. Cuylle, H. Linnartz, C. Jäger, and T. Henning, "A straightforward method for vacuum-ultraviolet flux measurements: The case of the hydrogen discharge lamp and implications for solid-phase actinometry," Appl. Phys. Lett. 105(1), 014105 (2014).

${ }^{42}$ S. E. Stein, "Mass spectra," in NIST Chemistry WebBook, NIST Standard Reference Database Number 69, edited by P. J. Lindstrom and W. G. Mallard (National Institute of Standards and Technology, 2018).

${ }^{43}$ G. A. Baratta and M. E. Palumbo, "Infrared optical constants of CO and $\mathrm{CO}_{2}$ thin icy films," J. Opt. Soc. Am. A 15, 3076-3085 (1998).

${ }^{44}$ Z. Dohnálek, G. A. Kimmel, P. Ayotte, R. S. Smith, and B. D. Kay, "The deposition angle-dependent density of amorphous solid water films," J. Chem. Phys. 118, 364-372 (2003).

${ }^{45}$ J.-B. Bossa, K. Isokoski, D. M. Paardekooper, M. Bonnin, E. P. van der Linden, T. Triemstra, S. Cazaux, A. G. G. M. Tielens, and H. Linnartz, "Porosity measurements of interstellar ice mixtures using optical laser interference and extended effective medium approximations," Astron. Astrophys. 561, A136 (2014).

${ }^{46}$ S. G. Warren and R. E. Brandt, "Optical constants of ice from the ultraviolet to the microwave: A revised compilation," J. Geophys. Res. 113(D14), D14220, https://doi.org/10.1029/2007JD009744 (2008).

${ }^{47}$ M. Bouilloud, N. Fray, Y. Bénilan, H. Cottin, M.-C. Gazeau, and A. Jolly, "Bibliographic review and new measurements of the infrared band strengths of pure molecules at $25 \mathrm{~K}: \mathrm{H}_{2} \mathrm{O}, \mathrm{CO}_{2}, \mathrm{CO}, \mathrm{CH}_{4}, \mathrm{NH}_{3}, \mathrm{CH}_{3} \mathrm{OH}, \mathrm{HCOOH}$ and $\mathrm{H}_{2} \mathrm{CO}$," Mon. Not. R. Astron. Soc. 451, 2145-2160 (2015).

${ }^{48}$ H. G. Kjaergaard, T. W. Robinson, and K. A. Brooking, "Calculated CHstretching overtone spectra of naphthalene, anthracene and their cations," J. Phys. Chem. A 104, 11297-11303 (2000).

${ }^{49}$ J. Malkin, Photophysical and Photochemical Properties of Aromatic Compounds (CRC Press, 1992).

${ }^{50}$ D. L. Kokkin, N. J. Reilly, T. P. Troy, K. Nauta, and T. W. Schmidt, "Gas phase spectra of all-benzenoid polycyclic aromatic hydrocarbons: Triphenylene," J. Chem. Phys. 126(8), 084304 (2007).
${ }^{51}$ V. Kofman, P. J. Sarre, R. E. Hibbins, I. L. ten Kate, and H. Linnartz, "Laboratory spectroscopy and astronomical significance of the fullybenzenoid PAH triphenylene and its cation," Mol. Astrophys. 7, 19-26 (2017).

${ }^{52}$ T. Keszthelyi, G. Balakrishnan, R. Wilbrandt, W. A. Yee, and F. Negri, "Evidence of dynamical Jahn-Teller effect on triphenylene radical cation: Resonance Raman spectrum and ab initio quantum-chemical calculations," J. Phys. Chem. A 104, 9121-9129 (2000).

${ }^{53}$ S. R. Langhoff, "Theoretical infrared spectra for polycyclic aromatic hydrocarbon neutrals, cations, and anions," J. Chem. Phys. 100(8), 2819-2841 (1996).

${ }^{54}$ C. Boersma, C. W. Bauschlicher, Jr., A. Ricca, A. L. Mattioda, J. Cami, E. Peeters, F. Sánchez de Armas, G. Puerta Saborido, D. M. Hudgins, and L. J. Allamandola, "The NASA Ames PAH IR spectroscopic database version 2.00: Updated content, web site, and on(off)line tools," Astrophys. J., Suppl. Ser. 211, 8 (2014).

${ }^{55}$ C. W. Bauschlicher, Jr., A. Ricca, C. Boersma, and L. J. Allamandola, "The NASA Ames PAH IR spectroscopic database: Computational version 3.00 with updated content and the introduction of multiple scaling factors," Astrophys. J., Suppl. Ser. 234, 32 (2018).

${ }^{56}$ E. E. Hardegree-Ullman, M. S. Gudipati, A. C. A. Boogert, H. Lignell, L. J. Allamandola, K. R. Stapelfeldt, and M. Werner, "Laboratory determination of the infrared band strengths of pyrene frozen in water ice: Implications for the composition of interstellar ices,” Astrophys. J. 784, 172 (2014).

${ }^{57}$ J. Bouwman, A. L. Mattioda, H. Linnartz, and L. J. Allamandola, "Photochemistry of polycyclic aromatic hydrocarbons in cosmic water ice. I. Mid-IR spectroscopy and photoproducts," Astron. Astrophys. 525, A93 (2011).

${ }^{58}$ M. P. Bernstein, S. A. Sandford, and L. J. Allamandola, "The infrared spectra of nitriles and related compounds frozen in $\mathrm{Ar}$ and $\mathrm{H}_{2} \mathrm{O}$, ” Astrophys. J. 476 , 932-942 (1997).

${ }^{59}$ K. Altwegg, H. Balsiger, A. Bar-Nun, J.-J. Berthelier, A. Bieler, P. Bochsler, C. Briois, U. Calmonte, M. R. Combi, H. Cottin, J. De Keyser, F. Dhooghe, B. Fiethe, S. A. Fuselier, S. Gasc, T. I. Gombosi, K. C. Hansen, M. Haessig, A. Ja ckel, E. Kopp, A. Korth, L. Le Roy, U. Mall, B. Marty, O. Mousis, T. Owen, H. Reme, M. Rubin, T. Semon, C.-Y. Tzou, J. H. Waite, and P. Wurz, "Prebiotic chemicals-amino acid and phosphorus-in the coma of comet 67P/Churyumov-Gerasimenko," Sci. Adv. 2, e1600285 (2016).

${ }^{60}$ P. Ehrenfreund, M. P. Bernstein, J. P. Dworkin, S. A. Sandford, and L. J. Allamandola, "The photostability of amino acids in space," Astrophys. J. 550, L95-L99 (2001).

${ }^{61}$ L. L. Danylewych and R. W Nicholls, "Intensity measurements and transition probabilities for bands of the $\mathrm{CN}$ violet $\left(\mathrm{B}^{2} \Sigma-\mathrm{X}^{2} \Sigma\right)$ band system," Proc. R. Soc. A 360, 557-573 (1978). 